\title{
Stem Cells in Wound Healing: The Future of Regenerative Medicine? A Mini-Review
}

\author{
Dominik Duscher $^{a} \quad$ Janos Barrera ${ }^{a}$ Victor W. Wong ${ }^{c}$ Zeshaan N. Maan ${ }^{a}$ \\ Alexander J. Whittam ${ }^{a}$ Michael Januszyk ${ }^{a, b}$ Geoffrey C. Gurtner ${ }^{a}$ \\ a Division of Plastic and Reconstructive Surgery, Department of Surgery, and b Program in Biomedical Informatics, \\ Stanford University School of Medicine, Stanford, Calif., and ' Department of Plastic Surgery, Johns Hopkins \\ University School of Medicine, Baltimore, Md. USA
}

\section{Key Words}

Stem cells · Progenitor cells · Wound healing · Cutaneous regeneration · Aging

\begin{abstract}
The increased risk of disease and decreased capacity to respond to tissue insult in the setting of aging results from complex changes in homeostatic mechanisms, including the regulation of oxidative stress and cellular heterogeneity. In aged skin, the healing capacity is markedly diminished resulting in a high risk for chronic wounds. Stem cell-based therapies have the potential to enhance cutaneous regeneration, largely through trophic and paracrine activity. Candidate cell populations for therapeutic application include adult mesenchymal stem cells, embryonic stem cells and induced pluripotent stem cells. Autologous cell-based approaches are ideal to minimize immune rejection but may be limited by the declining cellular function associated with aging. One strategy to overcome age-related impairments in various stem cell populations is to identify and enrich with functionally superior stem cell subsets via single cell transcriptomics. Another approach is to optimize cell delivery to the harsh environment of aged wounds via scaffold-based cell applications to enhance engraftment and paracrine activity of therapeutic stem cells. In this review, we shed light
\end{abstract}

on challenges and recent advances surrounding stem cell therapies for wound healing and discuss limitations for their clinical adoption.

(c) 2015 S. Karger AG, Basel

\section{Introduction}

Aging is a complex, multifactorial process characterized by an increased susceptibility to disease and tissue dysfunction [1]. Concomitant with advanced age is an increased risk for cardiovascular disease, peripheral vascular disease, and impaired wound healing [2]. Comorbidities such as diabetes further hinder the normal wound healing process, and increase the risk for chronic wound development in the elderly [3]. Defects in new blood vessel formation (neovascularization) have been identified as one of the main underlying causes [4].

Treatment strategies for nonhealing wounds in the older patient include addressing systemic factors (e.g. correcting blood sugar and optimizing cardiac output and blood oxygenation), providing local wound care through dressing changes and bedside or operative de-

\section{KARGER}

E-Mail karger@karger.com

www.karger.com/ger
(C) 2015 S. Karger AG, Basel

0304-324X/15/0622-0216\$39.50/0
Geoffrey C. Gurtner, MD, FACS

Division of Plastic and Reconstructive Surgery, Department of Surgery

Stanford University School of Medicine, 257 Campus Drive West

Hagey Building GK-201, Stanford, CA 94305-5148 (USA)

E-Mail ggurtner@stanford.edu 
bridement, administering antibiotics in the setting of infection, and, when conservative therapy fails, reconstructive surgery using tissue grafts and flaps [5]. Despite a multitude of therapeutic options, chronic wound care has met only moderate clinical success, and treatment options are increasingly limited in older patients, who are often less able to tolerate surgical intervention [6].

Due to a variety of medical and epidemiological factors, we are facing a rapid increase in the elderly population. According to the latest projections, the United States population aged 65 and older is expected to more than double between 2012 and 2060 [7]. Currently, chronic wounds affect 6.5 million patients and cost in excess of USD 25 billion annually in the United States alone [8]. The significant social and economic burden represented by 'problem wounds' in the increasing elderly population has precipitated study of the mechanisms underlying cutaneous regeneration.

As a result, multiple new therapies for chronic wounds are currently being assessed in preclinical and clinical studies. Among these new treatment modalities, stem cell-based therapies have gained interest as a promising approach to enhance tissue regeneration. Despite recent first licenses by regulatory agencies in Canada [9] and Europe [10] for other indications, and a thriving clinical trial landscape around the world with Asia as the current frontrunner [11], no stem cell therapy for wound healing applications has yet been approved [12]. Major barriers to clinical translation of stem cell-based therapies, in addition to the potential risk of immunogenicity and tumorigenicity, include challenges in identifying the stem cell populations best suited for wound therapy, as well as developing optimized delivery vehicles and/or adjunctive therapies to protect and improve the regenerative capacity of stem cells within the wound environment.

In this review, we summarize the key properties of major stem cell populations with potential for use in cutaneous wound healing, including their origin, function, and therapeutic capacity. We additionally review current challenges to clinical implementation, and present several key developing technologies that may address these obstacles.

\section{Stem Cell Populations for Cutaneous Repair}

Stem cells are characterized by their multipotency and capacity for self-renewal [13]. Their therapeutic potential is largely due to their ability to secrete proregenerative cytokines, making them an attractive option for the treat- ment of chronic wounds [14]. Stem cells from numerous sources are currently being tested in preclinical and clinical trials for their ability to foster wound healing and tissue regeneration (table 1 ). These trials have not only proven autologous stem cell therapy to be safely tolerated [15-17], but also demonstrated positive clinical outcomes. For example, Procházka et al. [18] showed in a study of 96 patients with critical limb ischemia that treatment with autologous mesenchymal stem cells (MSCs) could reduce the frequency of major limb amputation, as compared to standard treatment, within the first 120 days of follow-up. A detailed discussion of the global clinical trial landscape for stem cell-based therapies is beyond the scope of this review, but we refer the reader to an excellent paper on the topic [11].

In this section, we give an overview of the origin, function, and therapeutic capacity of the most promising stem cell populations for wound healing applications: adult MSCs, embryonic stem cells (ESCs), and induced pluripotent stem cells (iPSCs; fig. 1).

\section{Mesenchymal Stem Cells}

According to the International Society of Cellular Therapy (ISCT), MSCs are defined by their ability to adhere to a plastic surface, by their expression of the surface markers CD73, CD90, and CD105, by their lack of expression of hematopoietic markers CD14, CD34, CD45, CD11b/CD79, and CD19/HLA-DR, and by their ability to differentiate along osteoblastic, adipocytic and chondrocytic pathways [19]. Isolated from tissues including bone marrow, adipose tissue, umbilical cord blood, nerve tissue, and dermis, MSCs have been administered both systemically [20] and locally $[14,21]$ for the treatment of cutaneous wounds.

Although MSCs have been shown to exhibit low levels of long-term incorporation into healing wounds, a growing body of research suggests that their therapeutic benefit is attributed to their release of trophic mediators, rather than a direct structural contribution [14,21]. Through the release of vascular endothelial growth factor (VEGF), stromal cell-derived factor-1, epidermal growth factor, keratinocyte growth factor, insulin-like growth factor, and matrix metalloproteinase-9, MSCs promote new vessel formation, recruit endogenous progenitor cells, and direct cell differentiation, proliferation, and extracellular matrix formation during wound repair $[14,22$, 23]. MSCs also exhibit key immunomodulatory properties though the secretion of interferon- $\lambda$, tumor necrosis factor- $\alpha$, interleukin- $1 \alpha$ and interleukin- $1 \beta$, as well as through the activation of inducible nitric oxide synthase 
Table 1. Clinical trials of stem cell-based therapies for wound healing

\begin{tabular}{|c|c|c|c|c|c|c|}
\hline Conditions & Interventions & Age & Phase & $\mathrm{n}$ & Status & Reference \\
\hline Nonhealing wounds & $\begin{array}{l}\text { Autologous BM-MSC/ } \\
\text { fibrin spray }\end{array}$ & $\geq 18$ & 1 & 66 & Active, not recruiting & $\begin{array}{l}\text { https://clinicaltrials.gov/ } \\
\text { ct2/show/NCT01751282 }\end{array}$ \\
\hline Diabetic foot & $\begin{array}{l}\text { Autologous BM-MSC } \\
\text { intramuscular/ } \\
\text { intra-arterial injection }\end{array}$ & $18-80$ & 2 & 30 & $\begin{array}{l}\text { Completed; safely } \\
\text { tolerated, wound } \\
\text { healing observed }\end{array}$ & $\begin{array}{l}\text { https://clinicaltrials.gov/ } \\
\text { ct2/show/NCT01065337 }\end{array}$ \\
\hline Diabetic ulcer & $\begin{array}{l}\text { Allogeneic BM-MSC } \\
\text { injection }\end{array}$ & $18-81$ & 1 and 2 & 10 & Not yet recruiting & $\begin{array}{l}\text { https://clinicaltrials.gov/ } \\
\text { ct2/show/NCT01686139 }\end{array}$ \\
\hline Critical limb ischemia & $\begin{array}{l}\text { Autologous CD34+ cell } \\
\text { injection }\end{array}$ & $21-80$ & 1 and 2 & 28 & $\begin{array}{l}\text { Completed; safely } \\
\text { tolerated, trends } \\
\text { toward limb salvage } \\
\text { with therapy observed }\end{array}$ & $\begin{array}{l}\text { https://clinicaltrials.gov/ } \\
\text { ct2/show/NCT00616980 }\end{array}$ \\
\hline Leg ulcer/gangrene & $\begin{array}{l}\text { Autologous peripheral blood } \\
\text { CD34+ cell injection }\end{array}$ & $20-80$ & 1 and 2 & 15 & $\begin{array}{l}\text { Completed; safely } \\
\text { tolerated, positive } \\
\text { trends in efficacy } \\
\text { parameters observed }\end{array}$ & $\begin{array}{l}\text { https://clinicaltrials.gov/ } \\
\text { ct2/show/NCT00221143 }\end{array}$ \\
\hline $\begin{array}{l}\text { Critical lower limb } \\
\text { ischemia }\end{array}$ & $\begin{array}{l}\text { Autologous BM-MNC } \\
\text { injection }\end{array}$ & $18-75$ & 1 and 2 & 60 & Not yet recruiting & $\begin{array}{l}\text { https://clinicaltrials.gov/ } \\
\text { ct2/show/NCT01903044 }\end{array}$ \\
\hline $\begin{array}{l}\text { Chronic venous } \\
\text { leg ulcer }\end{array}$ & $\begin{array}{l}\text { Autologous BMDC } \\
\text { implantation }\end{array}$ & $40-75$ & 1 & 4 & Active, not recruiting & $\begin{array}{l}\text { https://clinicaltrials.gov/ } \\
\text { ct2/show/NCT01750749 }\end{array}$ \\
\hline Chronic wound & Autologous ASC injection & $\geq 18$ & 2 & 25 & Recruiting & $\begin{array}{l}\text { https://clinicaltrials.gov/ } \\
\text { ct2/show/NCT02092870 }\end{array}$ \\
\hline
\end{tabular}

$\mathrm{BMAC}=$ Bone marrow aspiration concentrate; $\mathrm{BM}-\mathrm{MNC}=$ bone marrow-derived mononuclear cell; $\mathrm{BMDC}=$ bone marrow-derived cells; $\mathrm{hUCMSC}=$ human umbilical cord-derived mesenchymal stem cells.

[24]. MSC secretion of prostaglandin $\mathrm{E}_{2}$ further regulates fibrosis and inflammation, promoting tissue healing with reduced scarring [25]. Finally, MSCs display bactericidal properties through the secretion of antimicrobial factors and by upregulating bacterial killing and phagocytosis by immune cells [26].

\section{Bone Marrow-Derived MSCs}

First isolated in 1966 by Friedenstein et al. [27], bone marrow-derived MSCs (BM-MSCs) are normally harvested from humans by aspiration from the iliac crest, followed by in vitro selection based on their ability to adhere to a plastic culture dish. Cells can then be expanded in culture and applied topically to wounds to promote tissue regeneration. Using normal and diabetic mice, $\mathrm{Wu}$ et al. [28] showed that injection of BM-MSCs into excisional wounds accelerates wound closure and increases re-epithelialization, angiogenesis, and cellularity. Clinical trials using alternate delivery methods have further confirmed the potential therapeutic efficacy of BM-MSCs in human cutaneous regeneration [22]. A study of human patients with chronic leg ulcers resistant to conventional treatment for at least 1 year showed that application of autologous BM-MSCs impregnated in a collagen matrix led to reduced wound size, increased vascularity, and increased dermal thickness [22]. More recently, a study of patients with either acute surgical or chronic lower extremity wounds found that application of BM-MSCs within a top- 
Fig. 1. Stem cell sources. Progenitor cells for wound therapies can be obtained from various sources, each with its particular advantages and disadvantages for clinical translation.

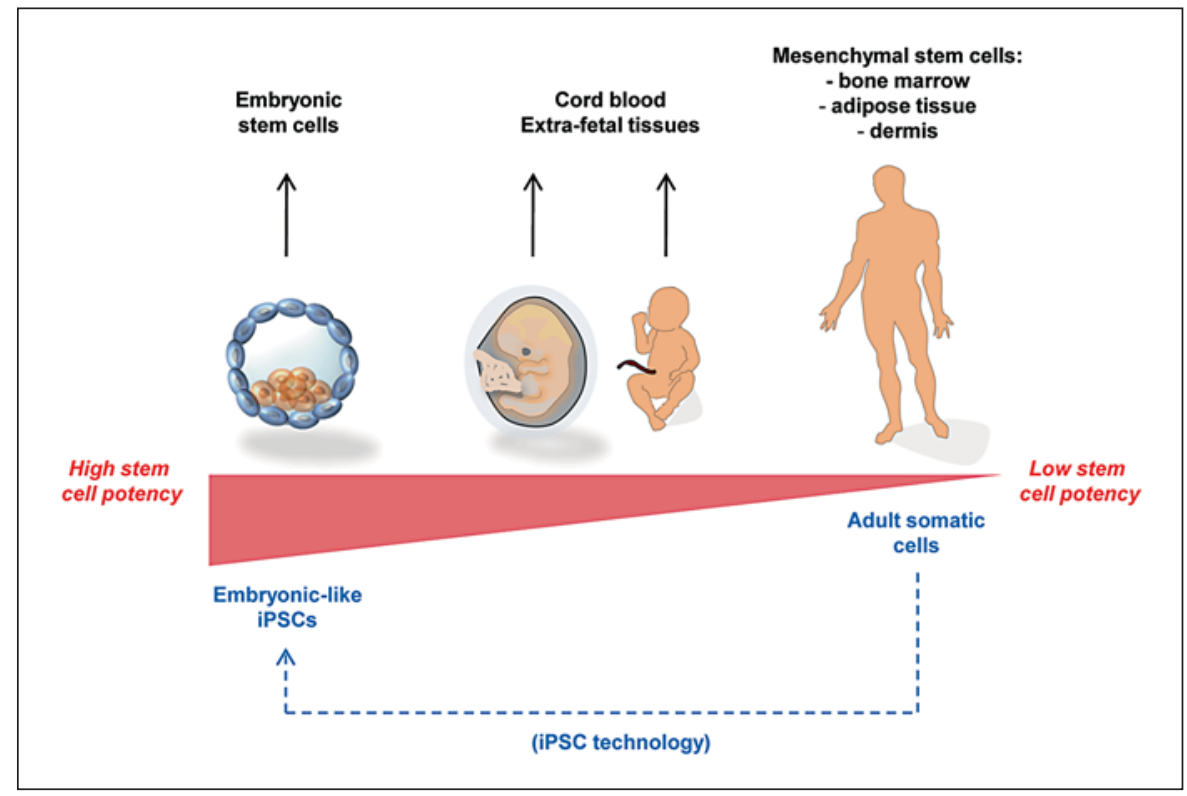

ical fibrin polymer spray led to a significant reduction in wound size [21]. However, significant challenges remain for the clinical translation of BM-MSCs, including the invasiveness of their harvest, as well as the time and resource costs and risks associated with expanding cells in culture in order to achieve therapeutic concentrations.

\section{Adipose-Derived MSCs}

Adipose-derived MSCs (ASCs) are a pluripotent, heterogeneous population of cells present within human adipose tissue with characteristics similar to BM-MSCs [29]. However, isolation of ASCs is readily accomplished using liposuction aspirates or excised fat samples, which are obtainable with minimal donor morbidity. Harvested fat is digested enzymatically with collagenase, followed by centrifugation, re-suspension and plating of the stromal vascular fraction for expansion [29] (fig. 2a). As with other MSCs, these cells adhere to plastic and are easily isolated and expanded in culture. ASCs can be differentiated into adipogenic, chondrogenic, myogenic, and osteogenic cell lineages in response to specific stimuli [30]. Alternatively, ASCs may be immediately administered without in vitro expansion or differentiation in culture. The extraordinarily high cell yield from lipoaspirate (as many as $1 \times 10^{7}$ cells from $300 \mathrm{ml}$ of lipoaspirate with at least $95 \%$ purity), as compared with bone marrow aspiration, makes ASCs a particularly attractive cell source for the acute wound setting [31].

ASCs have been tested in multiple preclinical trials on wound healing and have been found to significantly en- hance cutaneous wound healing and increase blood vessel formation $[14,32]$. A 2007 study showed that treating the radiated skin of cancer patients with purified autologous lipoaspirate led to improvements on a clinical and histological level [33]. Additionally, ASC-enriched fat grafts show improved survival rates in humans [34] and a recent phase I clinical trial confirmed the beneficial effects of ASCs in the treatment of critical limb ischemia [35]. Despite their increasing popularity, more clinical evidence is needed to substantiate a role for ASCs in humans. However, autologous ASC transplantation has been proven safe and well tolerated by patients without evidence of malignant transformation over a period of 120 days [34].

\section{Cord Blood and Extra-Fetal Tissue}

Human umbilical cord blood is a rich source of hematopoietic stem cells for the treatment of many pediatric and adult diseases. Despite early controversy [36], more recent studies have conclusively shown that MSCs can also be isolated from cord blood and that cord-derived MSCs accelerate wound healing [37]. Meanwhile, extrafetal tissues, including amniotic fluid, Wharton's jelly, placental tissue, and umbilical cord-lining tissue represent alternative stem cell sources. Amniotic fluid has been shown to contain MSCs expressing oct-4, a marker of pluripotency [38], and multipotent MSCs have been isolated from Wharton's jelly [39] and placental tissue [40]. Additionally, umbilical cord epithelial cells display stem-like properties and are capable of forming stratified epitheli- 


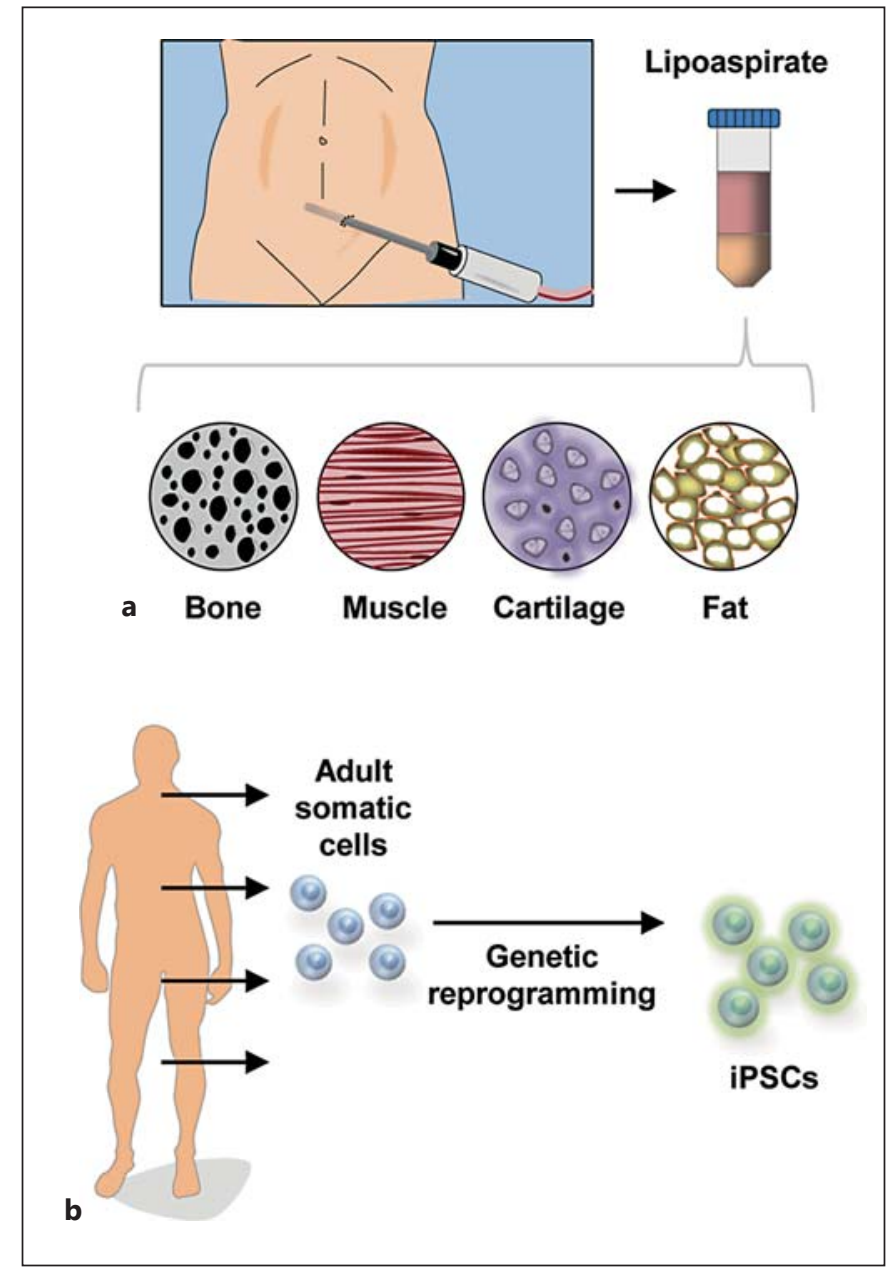

Fig. 2. Characteristics of different progenitor cell populations. a ASCs are routinely harvested from lipoaspirate and are multipotent with the capacity to differentiate down all four mesodermal lineages: fat, bone, cartilage, and muscle. After isolation they can be used therapeutically with and without ex vivo expansion. b Adult somatic cells can be reprogrammed as iPSCs with the same differentiation capacity as ESCs. With all their benefits and only a few of their limitations, iPSCs have significant advantages over ESCs, particularly with regard to clinical translation.

um in vitro and in vivo [41], representing a potential source for allogeneic skin grafts. Although extra-fetal tissue constitutes an abundant potential source of therapeutic stem cells, careful donor selection is critical to avoid immune-mediated rejection or transmission of genetic diseases, which may not be detected in the donor. Additional challenges include the need to develop efficient cell isolation, culture and expansion techniques and, as with most progenitor-based therapies, potential for malignant transformation in the long term.

\section{Skin Stem Cells}

MSCs have also been identified in all compartments of the skin. In the epidermis, there are 3 distinct stem cell populations responsible for the homeostasis of the superficial layers of the skin: interfollicular, sebaceous gland, and bulge area stem cells [42]. In the dermis, two MSC populations have been described so far: stem cells from the dermal papilla of hair follicles and a population of perivascular stem cells [42]. While cutaneous MSCs are less appealing as a source for cell-based therapeutics for wound healing applications, their contribution to local skin repair is still significant [43]. Manipulation and stimulation of these local progenitor cell populations via sophisticated biomaterial-based approaches that can precisely regulate spatial and temporal cues that define a functional niche environment could lead to efficacious treatment strategies to enhance wound healing and skin regeneration.

\section{Embryonic and Induced Pluripotent Stem Cells}

ESCs are pluripotent cells derived from the inner cell mass of the blastocyst and are capable of forming a complete organism [44]. In an attempt to utilize the remarkable regenerative potential of ESCs for cutaneous repair, Guenou et al. [45] differentiated ESCs into fully functional keratinocytes, which were subsequently used for reconstitution of the epidermis. Despite these promising findings, widespread clinical use of ESCs is currently elusive due to the potential for immunogenicity and tumorigenicity [46]. Additionally, ethical controversy and substantial legal restrictions surround the use of ESCs in any capacity. Although exciting progress has been made in harnessing the pluripotency of ESCs, regulatory issues and problems derived from their vast differentiation capacity must be addressed before clinical application becomes feasible.

A new class of stem cells, which potentially combines the advantages of MSCs and ESCs, has recently been discovered, ushering in a new era of regenerative medicine. Takahashi and Yamanaka [47] generated iPSC by reprogramming adult fibroblasts into an immature, pluripotent state (fig. 2b). The use of iPSC technologies allows for the generation of autologous pluripotent stem cell populations derived from differentiated adult tissues, thereby avoiding the ethical issues associated with human ESCs. Furthermore, autologous iPSCs are nonimmunogenic. Taking advantage of these characteristics, Itoh et al. [48] generated in vitro 3-D skin equivalents composed exclusively of human iPSC-derived keratinocytes and fibroblasts. Two recent studies have further proven this concept; Sebastiano et al. [49] and Umegaki-Arao et al. [50] 
Fig. 3. Challenges of the aged wound microenvironment. Several important characteristics set aged wounds apart from their younger counterparts. In advanced age, a progressive impairment of native stem cells and neovascularization have been identified as potential underlying causes of poor wound healing.

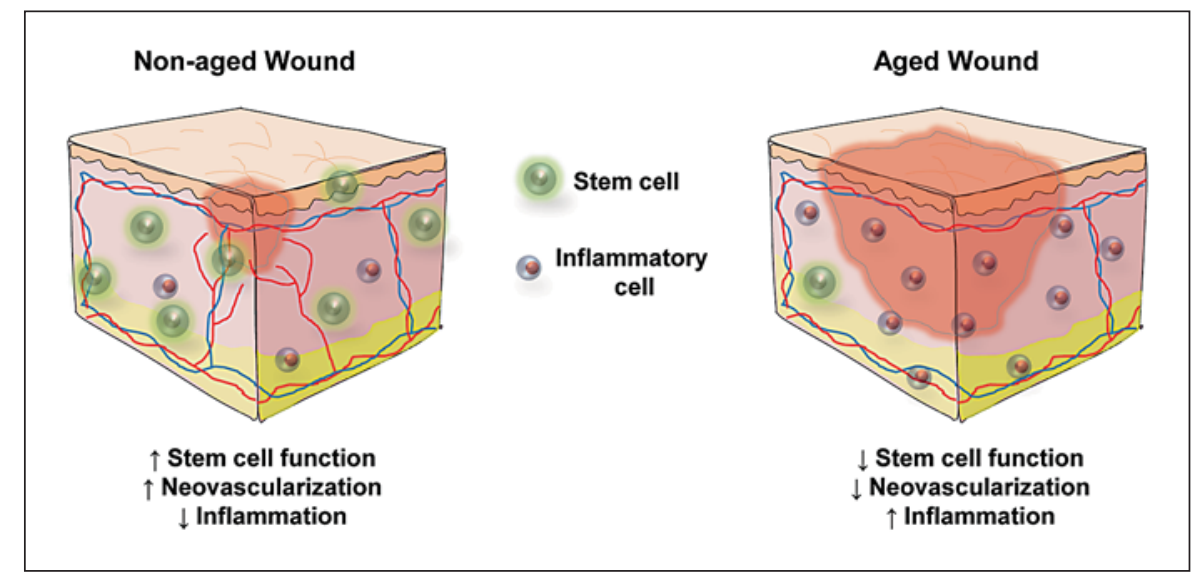

successfully used human keratinocyte-derived iPSCs to reconstitute skin in vitro for the treatment of recessive dystrophic epidermolysis bullosa. Additionally, Yang et al. [51] successfully differentiated iPSCs into folliculogenic human epithelial stem cells to regenerate all components of the hair follicle. These findings could enable the iPSC-based generation of complete cutaneous equivalents that include epidermal appendages.

iPSCs represent the combined advantages of the pluripotency of ESCs and the availability of MSCs. However, there are still numerous issues, such as their risk for tumorigenicity in an undifferentiated state, that need to be resolved before widespread clinical adaption is possible. Despite promising breakthroughs like the recent initiation of the first iPSC-based clinical trial for age-related macular degradation in Japan [52], iPSC-based therapies for wound healing applications require the improvement of their safety profile and refinement of current methods for differentiation into fibroblasts, keratinocytes, and associated cells with a focus on efficiency and cost-effectiveness.

\section{Challenges and Potential Solutions for Stem Cell Therapies in the Aged Wound}

The wound microenvironment, including the threedimensional extracellular matrix, and resident and recruited cells that secrete cytokines and various trophic and matrix-related factors, determines the capacity for tissue regeneration [53]. Systemic factors such as diabetes, vascular disease, and aging in particular, cause pathological changes to this microenvironment that must be fully understood in order to develop targeted therapies that restore the body's natural regenerative potential.
The cellular and tissue-level effects of aging have been studied extensively, although a fully comprehensive description of the aging process remains elusive. As this process relates to cutaneous healing physiology, several important characteristics set aged patients apart from their younger counterparts (fig. 3). In particular, progressive deficiencies in the function of native stem cells [54] and microvasculature [55], as well as the presence of a chronic systemic inflammatory state [56], likely play major contributory roles in poor wound healing in the aged setting and create difficult conditions for cell-based therapies. Apart from these age-specific factors, chronic wounds themselves are characterized by increased matrix metalloproteinase activity leading to aberrant tissue lysis and growth factor degradation [57]. Additionally, chronic wound fluid has been shown to impair stem cell proliferation and migration [58], representing further local challenges to stem cell therapy for cutaneous wounds. To overcome these hurdles and enable the use of stem cellbased therapies, our research team and others have been optimizing progenitor cell selection and delivery for chronic wounds in the aged patient.

\section{Microfluidic Single Cell Analysis to Enhance Stem Cell-Based Therapies}

Advanced age impairs the regenerative abilities of most tissues, and there is growing evidence that aged stem cells also suffer from decreased functionality, such as impaired differentiation capacity as well as alterations in therapeutic gene expression and cytokine production [59]. Nonetheless, these age-related differences remain incompletely understood, as do their functional consequences. In order to explore the mechanism by which aged stem cells fail to promote regeneration, we examined the impact of aging on progenitor cell ecology. We 
Fig. 4. Influence of advanced age on progenitor cell subpopulation dynamics. Biological aging manifests in a disruption of cellular subpopulation ecology.

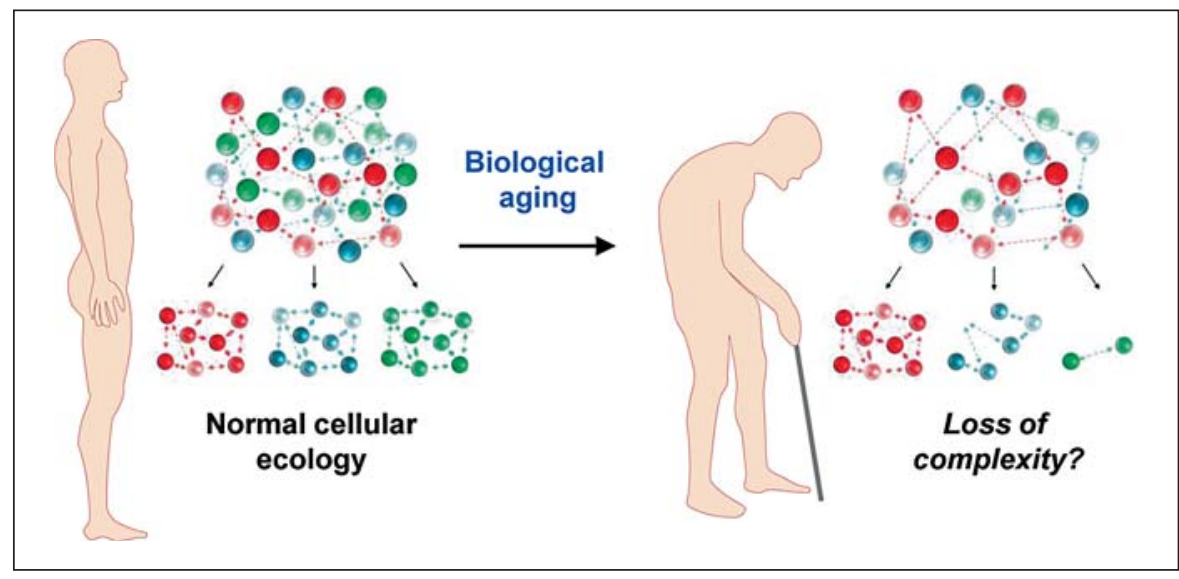

hypothesized that alterations in subpopulation distributions and loss of niche complexity underlie age-related stem cell impairments (fig. 4).

Integral to this work is the microfluidics-based single cell analytical approach developed within our laboratory, which combines single cell transcriptional probing with advanced statistical analysis [60]. Specifically, this technique utilizes quantitative PCR to simultaneously characterize 96 gene targets across hundreds of individual cells, allowing for higher-order correlations in expression patterns. We developed a novel computational methodology that applies principles of information theory and fuzzy logic to group cells based on their transcriptional signatures. In contrast to typical population-based analyses, this technique allows for the visualization of heterogeneity among individual cells, as well as the identification of distinct cell subsets.

Implementing this approach, we have recently found impaired neovascularization to be linked to the selective depletion of progenitor cell subpopulations in diabetes type 1 and 2 [61]. Given that diabetes and aging are both associated with dysfunctional vasculogenesis and share many cardiovascular sequelae, we thus hypothesized that a similar disruption in vascular progenitors would be associated with impaired neovascularization in aging. We employed our single cell analysis to examine ASCs and determine whether specific subgroups were altered or depleted in advanced age. High-resolution transcriptional analysis identified a subpopulation of ASCs that was considerably underrepresented in aged mice [59]. This potentially functionally relevant subset constitutes $61 \%$ of ASCs in young mice versus $39 \%$ in aged mice, demonstrating a deficiency in this stem cell population in aged subjects. This suggests disruption of the normal stem cell ecology as a result of aging (fig. 4). Bioinformatic charac- terization of this subgroup's expression profile highlighted a role for these cells in releasing proregenerative and antioxidative cytokines. For instance, aged ASC samples were depleted of a cellular subpopulation defined by increased expression of the master transcription factor hypoxia-inducible factor $1 a$ (Hifla) and the antioxidative enzyme superoxide dismutase 2 (Sod2). It has previously been shown that aged ASCs display reduced VEGF expression when cultured under hypoxic conditions [62]. Hifla is a direct upstream regulator of VEGF [63]. Our data demonstrating a depletion of a high Hifla-expressing subpopulation are consistent with a crucial role for this pathway in aged ASC dysfunction. As such, it may represent a potential target for ex vivo modulation prior to therapeutic application. Additionally, we identified an age-related decrease in ASCs expressing the antioxidative enzyme Sod2. Interestingly, while the presence of reactive oxygen species (ROS) has recently been linked to the improvement of aged ASC angiogenic potential [64], there is also strong evidence that ROS and oxidative stress directly inhibit angiogenesis [65]. These results suggest that a certain balance of oxidative stress is ideal and that aging may disrupt this balance and reduce the capacity of ASCs to modulate ROS levels within the wound and thereby reduce their therapeutic efficacy.

While the effectiveness of unselected autologous ASCs transplanted in aged patients is likely reduced, enrichment with specific proregenerative cell subpopulations may lead to improved ASC-based therapies in these patients. Therefore, after a shift of subpopulation dynamics in a disease state has been detected and a potent but reduced population has been identified, the next logical step is isolation of this functionally superior stem cell subset for therapeutic use. Since current cell sorting techniques are not mRNA-based but rather require a distinct surface 
Fig. 5. Stem cell delivery approaches for cutaneous wounds. A prerequisite for successful progenitor cell therapy for cutaneous wounds is an adequate delivery mechanism. Several different applications strategies with particular advantages and disadvantages are currently in preclinical and clinical evaluation, with bioscaffoldbased approaches appearing to be the most promising.

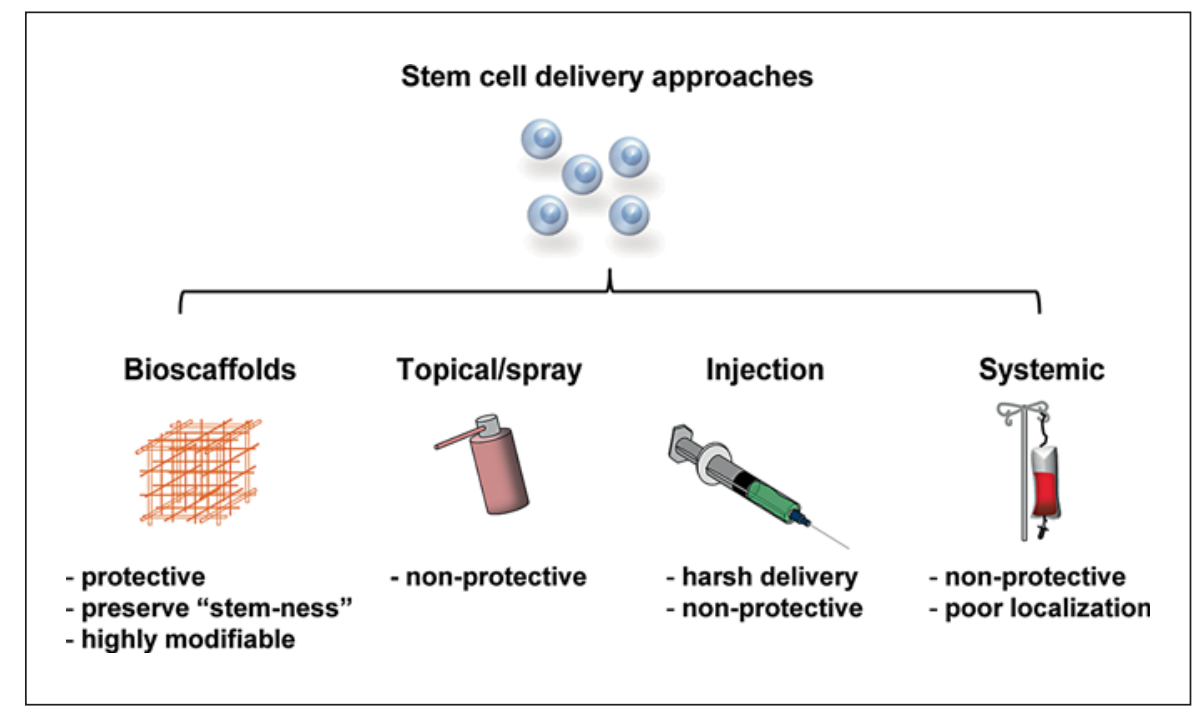

marker signature for identification, a correlation between the transcriptional signature of the desired cells with cell surface proteins is necessary. Linear discriminate analysis can be used to predict a protein-level association of gene expression profiles and surface markers [60]. Utilizing this method, we have been able to prospectively isolate desirable subpopulations of murine and human aged and diabetic ASCs via flow cytometry and assess them in vitro and in vivo with promising results regarding their regenerative abilities [Duscher et al., in preparation]. This novel approach provides a possibility to enhance the functionality of stem cells and will potentially make autologous cell-based therapeutics available to a broad patient cohort including aged and diabetic individuals.

\section{Novel Cell Delivery Approaches to Augment Cell}

Survival and Functionality

After a favorable progenitor population has been identified, cell delivery presents an additional challenge to clinical translation (fig. 5). Systemic administration is limited by a high cellular attrition rate and difficulties with tissue targeting. Therefore, local delivery has gained increasing acceptance as the optimal delivery approach for wound treatment. The therapeutic benefit of local MSC administration for the treatment of wounds has been well described in preclinical and early clinical studies $[14,21]$. However, hypoxia, oxidative stress and inflammation within the wound all contribute to a remarkably hostile environment for delivered cells. Cell engraftment following wound injection has been reported to be as low as $0 \%$ at 11 days in preclinical models, potentially from shear injury during injection [14]. Topical applica- tion (e.g. via spray) is limited by delivery of nonprotected cells into the wound environment as well as poor control of cell density and spacing. Additionally, maintaining engrafted cells in a 'stem-like' state versus accelerated cell differentiation may result in beneficial effects on wound healing, and warrants consideration when designing a delivery vehicle. In order to improve cell engraftment and therapeutic functionality, a number of alternative delivery mechanisms have been developed.

Bioscaffold-based delivery is an increasingly popular technique for stem cell transplantation. Composed of collagen, hyaluronic acid, or other naturally derived or synthetic materials, these matrices provide protection and controlled spatial cues for seeded stem cells in hopes of establishing a functional cell niche in addition to providing wound coverage. Our laboratory has shown that seeding stem cells onto a specialized bioscaffold composed of a pullulan-collagen matrix preserves cell expression of stemness-related genes and significantly accelerates wound healing compared to injection techniques [23]. We additionally showed that beyond basic mechanical protection, this pullulan-collagen bioscaffold is able to quench free radicals within ischemic wounds, thereby decreasing oxidative stress and increasing graft cell longevity [66]. To further improve the efficiency of topical progenitor cell therapy, novel approaches to scaffold seeding need to be established. Employing an innovative seeding methodology leveraging capillary force, ASCs could be seeded more effectively compared with established methods such as centrifugal, injection, and orbital seeding [14]. This represents an important step in readying stem cell-based therapies for clinical application. 
Another method for improving progenitor cell delivery and therapeutic efficacy is modifying the structure and composition of these matrices. Structural alteration of porous scaffolds offers a great degree of customizability for various progenitor cell therapeutics and wound settings. Recent evidence suggests that modifying the strength and character of intermolecular bonds within a scaffold can direct cell proliferation and differentiation behavior [67]. Aside from the scaffold itself, the addition of growth factors, small molecules, anti-inflammatory and/or antioxidant substances, plasmids, or other vectors may further modulate stem cell activity and wound healing. These 'smart' materials have remarkable potential for manipulating stem cells to further survival and regenerative activity beyond their normal physiological capacity. Techniques for material-based cell enhancement may represent a particularly attractive treatment strategy for aged patients, who may exhibit native stem cell deficiencies.

Despite recent advancements and first clinical trials, clinical adoption of stem cell-seeded scaffolds for cutaneous regeneration remains elusive. Existing limitations include safety, manufacturing costs, and mechanical integrity of the cell-scaffold system. Fortunately, several technological advancements for bioscaffold fabrication, such as laser-assisted and printer-based methods, enable rapid prototyping. Further translational testing and innovations arising from the intersection of engineering, biology, and medicine will eventually enable clinical application of this promising new treatment paradigm.

\section{Conclusion}

Cutaneous wounds in aged patients exhibit unique challenges requiring careful consideration when designing therapies for their repair. Native microvascular and inflammatory abnormalities in aged tissues present particular hindrances to adequate wound healing. Stem cells offer a promising approach to wound therapy in the aged setting through release of soluble growth factors and cytokines that stimulate new vessel formation and modulate inflammation. However, significant hurdles remain in optimizing progenitor cell selection and delivery. As our understanding of stem cell biology and heterogeneity grows, improved cell selection should lead to observable benefits. Emerging techniques such as microfluidic single cell characterization offer particularly attractive strategies for identifying and isolating the most effective cells for therapeutic use. Following selection of the most appropriate cell populations, effective delivery vehicles are needed to protect them within the wound environment and provide additional functional enhancement. Despite the multiple barriers to clinical implementation, stem cells have shown sufficient promise to garner a place in the field of regenerative medicine. Progenitor cell therapies have significant implications for human health. Clinical studies are greatly needed to build on recent successes in basic and translational research in hopes of revolutionizing wound care in the 21 st century.

\section{References}

1 Hayflick L: Biological aging is no longer an unsolved problem. Ann NY Acad Sci 2007; 1100:1-13.

-2 Reed MJ, Edelberg JM: Impaired angiogenesis in the aged. Sci Aging Knowledge Environ 2004;2004:pe7.

3 Rando TA: Stem cells, ageing and the quest for immortality. Nature 2006;441:1080-1086.

-4 Chang EI, et al: Age decreases endothelial progenitor cell recruitment through decreases in hypoxia-inducible factor 1alpha stabilization during ischemia. Circulation 2007;116: 2818-2829.

5 Werdin F, Tenenhaus M, Rennekampff HO: Chronic wound care. Lancet 2008;372:18601862.

6 Finlayson E, et al: Major abdominal surgery in nursing home residents: a national study. Ann Surg 2011;254:921-926.

7 US Census Bureau: Projections 2012-2060. Washington, US Census Bureau, 2012.
Sen CK, et al: Human skin wounds: a major and snowballing threat to public health and the economy. Wound Repair Regen 2009;17: 763-771.

9 Pollack A: A stem-cell-based drug gets approval in Canada. New York Times, May 17, 2012.

10 European Medicines Agency: First Stem-Cell Therapy Recommended for Approval in EU. London, European Medicines Agency, 2014.

11 Li MD, Atkins H, Bubela T: The global landscape of stem cell clinical trials. Regen Med 2014;9:27-39.

12 Rosemann A: Why regenerative stem cell medicine progresses slower than expected. J Cell Biochem 2014;115:2073-2076.

13 Behr B, et al: Stem cells. Plast Reconstr Surg 2010;126:1163-1171.

14 Garg RK, et al: Capillary force seeding of hydrogels for adipose-derived stem cell delivery in wounds. Stem Cells Transl Med 2014;3: 1079-1089.
15 Kirana S, et al: Autologous stem cell therapy in the treatment of limb ischaemia induced chronic tissue ulcers of diabetic foot patients. Int J Clin Pract 2012;66:384-393.

16 Kawamoto A, et al: Intramuscular transplantation of G-CSF-mobilized CD34+ cells in patients with critical limb ischemia: a phase I/IIa, multicenter, single-blinded, dose-escalation clinical trial. Stem Cells 2009;27:2857-2864.

17 Losordo DW, et al: A randomized, controlled pilot study of autologous CD34+ cell therapy for critical limb ischemia. Circ Cardiovasc Interv 2012;5:821-830.

18 Procházka V, et al: Cell therapy, a new standard in management of chronic critical limb ischemia and foot ulcer. Cell Transplant 2010; 19:1413-1424.

19 Dominici M, et al: Minimal criteria for defining multipotent mesenchymal stromal cells. The International Society for Cellular Therapy position statement. Cytotherapy 2006;8: 315-317. 
20 Caplan AI, Dennis JE: Mesenchymal stem cells as trophic mediators. J Cell Biochem 2006;98:1076-1084.

-21 Falanga V, et al: Autologous bone marrowderived cultured mesenchymal stem cells delivered in a fibrin spray accelerate healing in murine and human cutaneous wounds. Tissue Eng 2007;13:1299-1312.

$\checkmark 22$ Badiavas EV, Falanga V: Treatment of chronic wounds with bone marrow-derived cells. Arch Dermatol 2003;139:510-516.

23 Rustad KC, et al: Enhancement of mesenchymal stem cell angiogenic capacity and stemness by a biomimetic hydrogel scaffold. Biomaterials 2012;33:80-90.

-24 Ren G, et al: Mesenchymal stem cell-mediated immunosuppression occurs via concerted action of chemokines and nitric oxide. Cell Stem Cell 2008;2:141-150.

$25 \mathrm{Hu}$ MS, et al: The role of stem cells during scarless skin wound healing. Adv Wound Care 2014;3:304-314.

-26 Mei SHJ, et al: Mesenchymal stem cells reduce inflammation while enhancing bacterial clearance and improving survival in sepsis. Am J Respir Crit Care Med 2010;182:10471057.

27 Friedenstein AJ, Piatetzky-Shapiro II, Petrakova KV: Osteogenesis in transplants of bone marrow cells. J Embryol Exp Morphol 1966; 16:381-390.

28 Wu Y, et al: Mesenchymal stem cells enhance wound healing through differentiation and angiogenesis. Stem Cells 2007;25:2648-2659.

29 Zuk PA, et al: Multilineage cells from human adipose tissue: implications for cell-based therapies. Tissue Eng 2001;7:211-228.

-30 Zuk PA, et al: Human adipose tissue is a source of multipotent stem cells. Mol Biol Cell 2002;13:4279-4295.

31 Boquest AC, et al: Isolation of stromal stem cells from human adipose tissue. Methods Mol Biol 2006;325:35-46.

-32 Teng M, Huang Y, Zhang Y: Application of stems cells in wound healing - an update: stem cells in wound healing. Wound Repair Regen 2014;22:151-160.

-33 Rigotti G, et al: Clinical treatment of radiotherapy tissue damage by lipoaspirate transplant: a healing process mediated by adiposederived adult stem cells. Plast Reconstr Surg 2007;119:1409-1422; discussion 1423-1424.

34 Kolle SF, et al: Enrichment of autologous fat grafts with ex-vivo expanded adipose tissuederived stem cells for graft survival: a randomised placebo-controlled trial. Lancet 2013;382:1113-1120.

35 Bura A, et al: Phase I trial: the use of autologous cultured adipose-derived stroma/stem cells to treat patients with non-revascularizable critical limb ischemia. Cytotherapy 2014; $16: 245-257$.
36 Kamolz LP, et al: Cultured human epithelium: human umbilical cord blood stem cells differentiate into keratinocytes under in vitro conditions. Burns 2006;32:16-19.

37 Liao Y, et al: Human cord blood-derived unrestricted somatic stem cells promote wound healing and have therapeutic potential for patients with recessive dystrophic epidermolysis bullosa. Cell Transplant 2014;23:303-317.

38 In 't Anker PS, et al: Amniotic fluid as a novel source of mesenchymal stem cells for therapeutic transplantation. Blood 2003;102:15481549.

39 Kim W-S, et al: Wound healing effect of adipose-derived stem cells: a critical role of secretory factors on human dermal fibroblasts. J Dermatol Sci 2007;48:15-24.

40 Alviano F, et al: Term amniotic membrane is a high throughput source for multipotent mesenchymal stem cells with the ability to differentiate into endothelial cells in vitro. BMC Dev Biol 2007;7:11.

41 Sanmano B, et al: Engraftment of umbilical cord epithelial cells in athymic mice: in an attempt to improve reconstructed skin equivalents used as epithelial composite. J Dermatol Sci 2005;37:29-39.

42 Wong VW, et al: Stem cell niches for skin regeneration. Int J Biomater 2012;2012:926059.

43 Wong VW, Gurtner GC, Longaker MT: Wound healing: a paradigm for regeneration. Mayo Clin Proc 2013;88:1022-1031.

44 Odorico JS, Kaufman DS, Thomson JA: Multilineage differentiation from human embryonic stem cell lines. Stem Cells 2001;19:193204.

45 Guenou H, et al: Human embryonic stemcell derivatives for full reconstruction of the pluristratified epidermis: a preclinical study. Lancet 2009;374:1745-1753.

46 Wu DC, Boyd AS, Wood KJ: Embryonic stem cell transplantation: potential applicability in cell replacement therapy and regenerative medicine. Front Biosci 2007;12:4525-4535.

47 Takahashi K, Yamanaka S: Induction of pluripotent stem cells from mouse embryonic and adult fibroblast cultures by defined factors. Cell 2006;126:663-876.

48 Itoh $\mathrm{M}$, et al: Generation of 3D skin equivalents fully reconstituted from human induced pluripotent stem cells (iPSCs). PLoS One 2013;8:e77673.

49 Sebastiano V, et al: Human COL7A1-corrected induced pluripotent stem cells for the treatment of recessive dystrophic epidermolysis bullosa. Sci Transl Med 2014;6:264ra163.

50 Umegaki-Arao N, et al: Induced pluripotent stem cells from human revertant keratinocytes for the treatment of epidermolysis bullosa. Sci Transl Med 2014;6:264ra164.
51 Yang R, et al: Generation of folliculogenic human epithelial stem cells from induced pluripotent stem cells. Nat Commun 2014;5: 3071.

52 Reardon S: Cyranoski, Japan stem-cell trial stirs envy. Nature 2014;513:287-288.

53 Gurtner GC, et al: Wound repair and regeneration. Nature 2008;453:314-321.

54 Sharpless NE, DePinho RA: How stem cells age and why this makes us grow old. Nat Rev Mol Cell Biol 2007;8:703-713.

55 Lähteenvuo J, Rosenzweig A: Effects of aging on angiogenesis. Circ Res 2012;110:12521264.

56 Donato AJ, et al: Aging is associated with greater nuclear NF kappa B, reduced I kappa $B$ alpha, and increased expression of proinflammatory cytokines in vascular endothelial cells of healthy humans. Aging Cell 2008;7: 805-812.

57 Trengove NJ, et al: Analysis of the acute and chronic wound environments: the role of proteases and their inhibitors. Wound Repair Regen 1999;7:442-452.

58 Koenen P, et al: Acute and chronic wound fluids inversely influence adipose-derived stem cell function: molecular insights into impaired wound healing. Int Wound J 2015;12: $10-16$.

59 Duscher D, et al: Aging disrupts cell subpopulation dynamics and diminishes the function of mesenchymal stem cells. Sci Rep 2014;4: 7144.

60 Januszyk M, Gurtner GC: High-throughput single-cell analysis for wound healing applications. Adv Wound Care 2013;2:457-469.

61 Januszyk M, et al: Diabetes irreversibly depletes bone marrow-derived mesenchymal progenitor cell subpopulations. Diabetes 2014;63:3047-3056.

62 Efimenko A, et al: Angiogenic properties of aged adipose derived mesenchymal stem cells after hypoxic conditioning. J Transl Med 2011;9:10.

63 Duscher D, et al: Transdermal deferoxamine prevents pressure-induced diabetic ulcers. Proc Natl Acad Sci USA 2015;112:94-99.

64 De Barros S, et al: Aging-related decrease of human ASC angiogenic potential is reversed by hypoxia preconditioning through ROS production. Mol Ther 2013;21:399-408.

65 Perveen S, et al: Role of EC-SOD overexpression in preserving pulmonary angiogenesis inhibited by oxidative stress. PLoS One 2012; 7:e51945.

66 Wong VW, et al: Pullulan hydrogels improve mesenchymal stem cell delivery into high-oxidative-stress wounds. Macromol Biosci 2011; 11:1458-1466.

67 Jeon O, Alsberg E: Regulation of stem cell fate in a three-dimensional micropatterned dualcrosslinked hydrogel system. Adv Funct Mater 2013;23:4765-4775. 